\title{
Automatic system for optical parameters measurements of biological tissues
}

\author{
Paulina Listewnik, ${ }^{* 1}$ Adam Mazikowski, ${ }^{1}$ \\ ${ }^{1}$ Department of Metrology and Optoelectronics, Faculty of Electronics, Telecommunications and Informatics, \\ Gdańsk University of Technology, Narutowicza Street 11/12, 80-233 Gdańsk, Poland
}

Received September 09, 2018; accepted September 30, 2018; published September 30, 2018

\begin{abstract}
In this paper a system allowing execution of automatic measurements of optical parameters of scattering materials in an efficient and accurate manner is proposed and described. The system is designed especially for measurements of biological tissues including phantoms, which closely imitate optical characteristics of real tissue The system has modular construction and is based on the ISEL system, luminance and color meter and a computer with worked out dedicated software and user interface. Performed measurements of scattering distribution characteristics for selected materials revealed good accuracy, confirmed by comparative measurements using well-known reference characteristics.
\end{abstract}

Optical parameters of tissues play a very important role in light therapy or medical diagnostics performed by optical methods. Because of additional restriction and convenience reasons, a lot of preclinical research is conducted using phantoms, which closely imitate optical characteristics of real tissue. Because their parameters can be controlled, optical phantoms are useful in determining whether devised testing methods are adequate to be used on live tissue [1-3].

The scattering, absorption, and scattering anisotropy are the basic optical properties that describe the photon migration in human tissue [1]. Full characterization is usually obtained by determination of Bidirectional Scattering Distribution Function (BSDF) [4].

Manual measurements can be sufficient during singlepoint testing. However, during executing multiple related measurements, e.g. surface uniformity or spatial (angular) distribution, it is crucial to automatize the process to obtain reliable data.

Therefore, an automatic system for measurements of optical parameters of scattering materials (e.g. biological tissues or phantoms) was developed. The system ensures high accuracy and repeatability of measurements as well as mechanical stability of measurement configuration (e.g. avoiding displacement of a sample during the measurement process). A block diagram of the developed system is presented in Fig. 1.

\footnotetext{
*E-mail: paulist@o2.pl
}

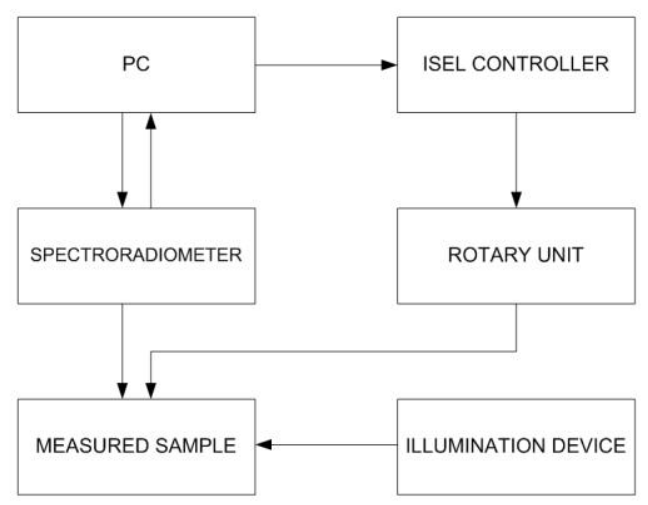

Fig. 1. Block diagram of the developed system.

The system consists of a main unit (computer with dedicated software), ISEL rotary unit with an ISEL controller (ISEL Germany AG), measuring device and illumination device.

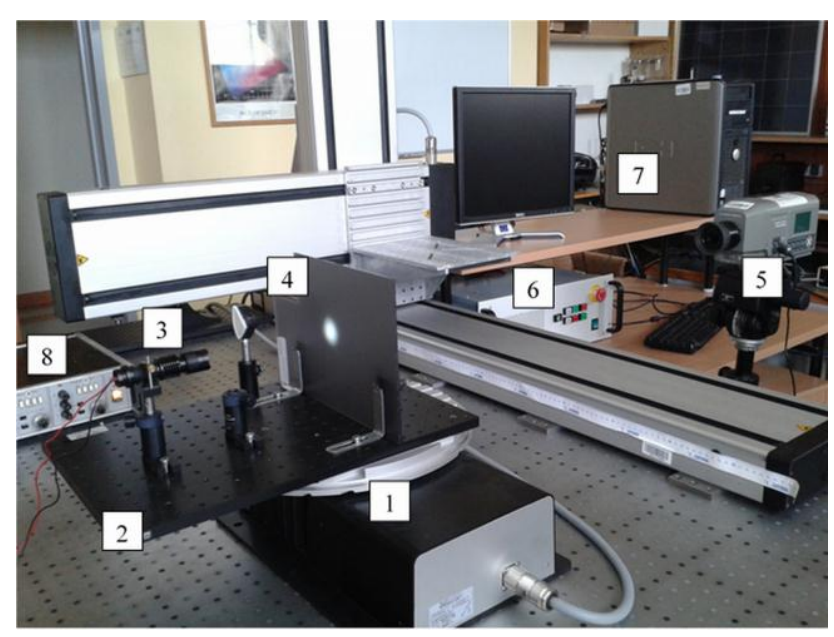

Fig. 2. View of the measurement setup: 1 - rotary unit, 2 - Thorlabs MB3045/M breadboard, 3 - illumination device, 4 - sample under test, 5 - measurement device, 6 - controller, 7 - computer, 8 - illumination device power supply.

An ISEL rotary unit (standard fitted with an aluminum Tgroove plate) was also equipped with a Thorlabs 
breadboard aluminum plate. Such a solution increases the flexibility of a measurement setup configuration and allows for a firm attachment of different samples to the plate (surface of the examined sample has to correspond to the axis of rotation) as well as the illumination unit. An ISEL rotary unit allows to shift the sample with angular accuracy up to $0.16^{\circ}$, each way (left and right) [5]. A view of the measurement setup is shown in Fig. 2.

As a measurement device, luminance and color meter Konica Minolta CS-200 (Konica Minolta Inc., Japan) was applied. It is set stationary (does not rotate) and allows for high accuracy spot measurements of luminance and color coordinates [6]. Replacing this meter by a more advanced model (e.g. spectroradiometer Konica Minolta CS-2000A) will also allow for the measurement of a spectrum.

Dedicated software for the whole system controlling was developed and implemented by the authors in LabView 12.0 (National Instruments, USA) and allows for communication between both the spectroradiometer and ISEL controller via an USB cable. First, the user has to calibrate the system and then introduce (manually or automatically) the measurement coordinates (angles). In the course of testing the measurement device transfers data back to the computer, where they are processed and displayed in a user-friendly manner. The color coordinates were marked on the CIE 1931 Yxy colorimetric diagram [7], while measured luminance was displayed as a function of angle. A view of the final report of performed measurements was presented in Fig. 3.

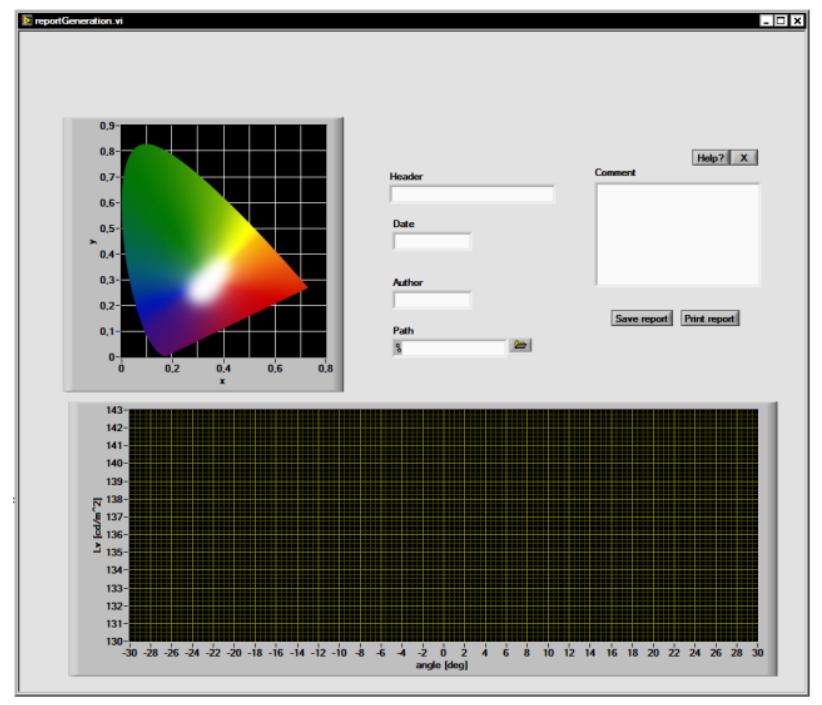

Fig. 3. View of the report window.

In order to test the correctness of the system's operation, measurements of spatial luminance distribution for several selected materials were carried out. Finally, four different materials were tested: samples of screens for back projection, a sheet of paper with a density and a diffuser made of two layers of tracing paper were used.

The screen samples for back projection (Plexiglas RP 99561 and Plexiglas RP 7D006 by Evonik Rohm GmbH, Germany) were mainly tested for their well-known directional scattering characteristics, provided by the manufacturer (Fig. 4).

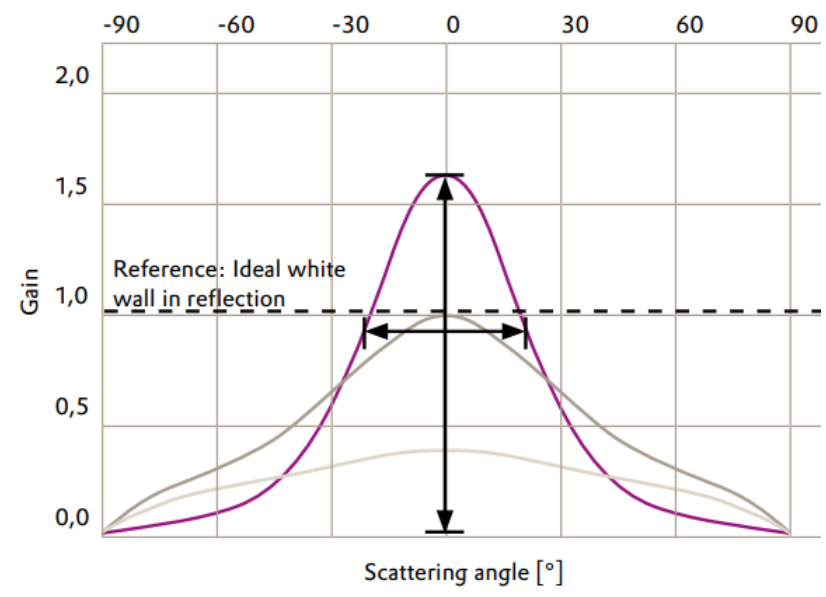

Fig. 4. Scattering characteristics of Plexiglas RP 99561, Plexiglas RP 7D007 and Pklexiglas RP 7D006 [8].

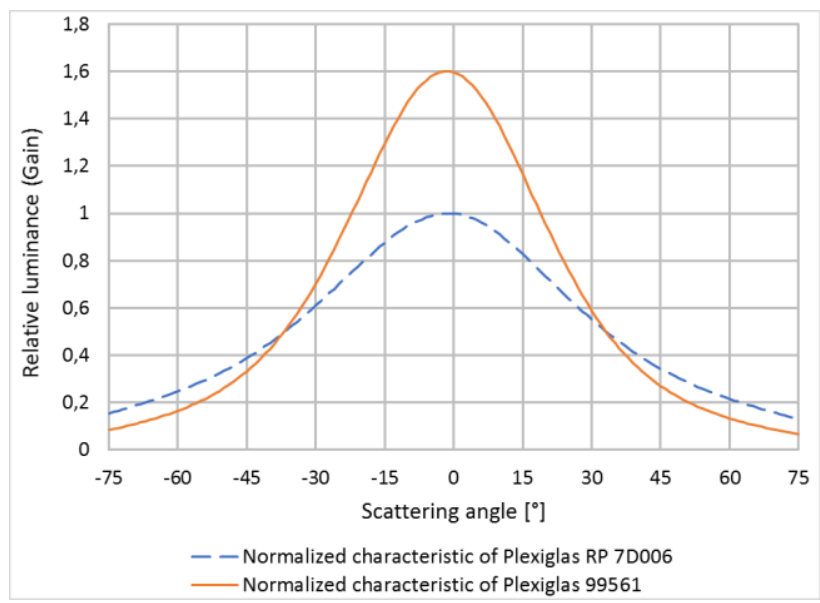

Fig. 5. Normalized measured characteristics of Plexiglas RP 99561 and Pklexiglas RP 7D006.

Both measured characteristics are slightly shifted to the left due to inaccurate setting of $0^{\circ}$ angle. However, as it can be noticed, a very good agreement between the measured and reference characteristics of luminance distribution was obtained. Additional measurements were performed for two different scattering layers: a white paper sheet (of density $80 \mathrm{~g} / \mathrm{m}^{2}$ ) and a diffuser made of two layers of tracing paper separated from each other by about 30mm. The results are presented in Fig. 6 and Fig. 7, respectively. Although the diffuser was made of two layers, its spatial characteristic is narrower than that 
of a single sheet of paper, so a single sheet of paper seems to be a very good diffuser. What is characteristic for a sheet of paper is mainly the fact that not being smooth, a single, thin sheet of paper is not forming a plane. It should be taken into account for further measurements of thin phantoms or tissues.

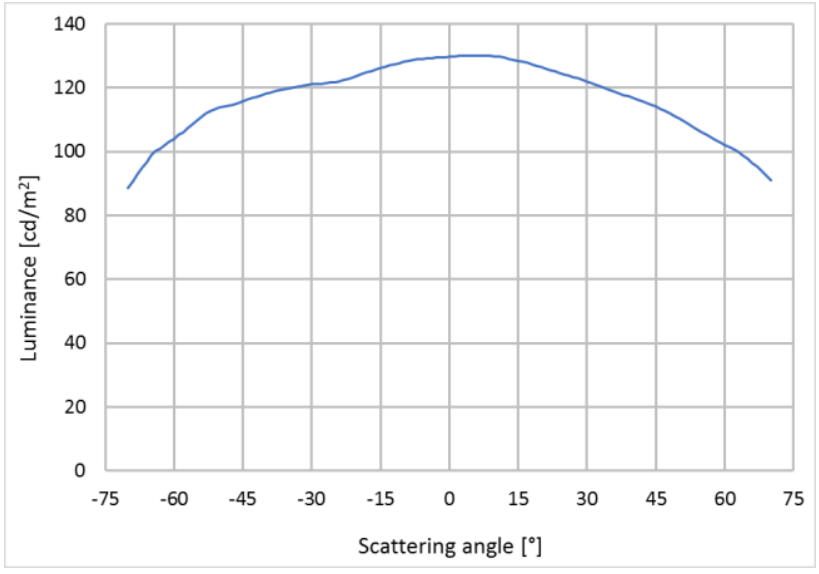

Fig. 6. Measured characteristics of the white paper sheet of a density of $80 \mathrm{~g} / \mathrm{m}^{2}$.

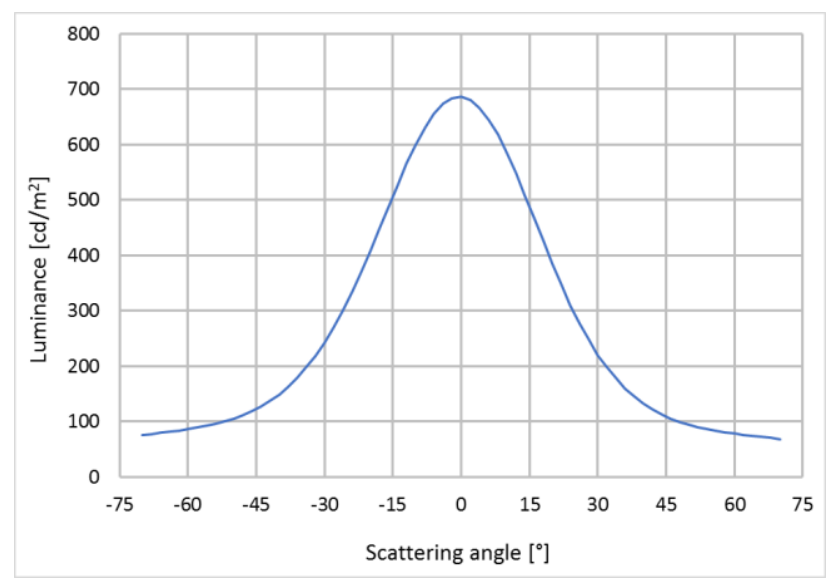

Fig. 7. Measured characteristics of a diffuser made of two layers of tracing paper separated from each other.

In conclusion, the developed measurement system allows for determination of spatial distribution of optical parameters. It is characterized by good accuracy, confirmed in comparative measurements, thus it can be a useful tool for measurement of optical properties of biological tissues or phantoms.
The authors gratefully acknowledge the financial support from the DS funds of the Faculty of Electronics, Telecommunications, and Informatics of the Gdańsk University of Technology.

The authors would also like to express great appreciation to Michał Meironke for his valuable and constructive help during the development of this work.

\section{References}

[1] M.S. Wróbel, A.P. Popov, A.V. Bykov, M. Kinnunen, M. Jedrzejewska-Szczerska, V.V. Tuchin, J. Biomed. Opt. 20(4), 045004 (2015). https://doi.org/10.1117/1.JBO.20.4.045004

[2] M.S. Wróbel, M. Jedrzejewska-Szczerska, S. Galla, L. Piechowski, M. Sawczak, A.P. Popov, A. Cenian, J. Biomed. Opt. 20(8), 085003 (2015). https://doi.org/10.1117/1.JBO.20.8.085003

[3] M. Jędrzejewska-Szczerska, M.S. Wróbel, S. Galla, A.P. Popov, A.V. Bykov, V.V. Tuchin, A. Cenian, Proc. SPIE 9447, 944715 (2015). https://doi.org/10.1117/12.2177790

[4] A.M. Brown et al., Proc. SPIE 7792, 7792211 (2010).

[5] 4-Axis Controller: iMC-S8. Operating Instruction (ISEL Germany AG, 2012).

[6] Konica Minolta, Inc. (2005-2013). Chroma meter CS-200. Datasheet.

[7] D. Malacara, Color Vision and Colorimetry; Theory and Applications (SPIE Press, 2002).

[8] A. Mazikowski, M. Trojanowski, Metrol. Measur. Systems 20(3), 443 (2013). 